\title{
A INTEGRALIDADE NO COTIDIANO DAS PRÁTICAS EM UM CENTRO DE ATENÇÃO PSICOSSOCIAL*
}

Denise Bermudez Pereira1, Valéria Cristina Christello Coimbra ${ }^{2}$, Luciane Prado Kantorski ${ }^{3}$, Michele Mandagará de Oliveira $^{4}$, Marilu Correa Soares ${ }^{4}$, Greice Schrader ${ }^{5}$

RESUMO: Estudo de caso descritivo, com abordagem qualitativa, realizado num Centro de Atenção Psicossocial I, ao sul do Estado do Rio Grande do Sul, teve por objetivo analisar o cotidiano das práticas dos profissionais e como referencial a integralidade na organização das práticas. Os dados foram coletados por meio de entrevista a 21 integrantes da equipe e, por meio da análise temática, foram desveladas as categorias Espaços de interação e construção: a integralidade nas práticas cotidianas e Dificuldades enfrentadas no cotidiano do serviço. Verificou-se que a lógica do serviço está constituída de maneira usuário-centrada; que espaços de interação e construção propiciam a integração entre equipe e usuários, potencializando a integralidade. Apesar de algumas dificuldades, como a existência do ambulatório de saúde mental dentro do serviço, concluiu-se que as práticas vão ao encontro da integralidade, já que se propõem à construção de novas alternativas de cuidado, valorizando o ser humano em sofrimento.

PALAVRAS-CHAVE: Assistência integral à saúde; Saúde mental; Serviços de saúde.

\section{INTEGRALITY IN THE ROUTINES OF PRACTICE IN A PSYCHO-SOCIAL ATTENTION CENTER}

\begin{abstract}
This descriptive case study with a qualitative approach, carried out in a Center for Psycho-Social Attention in the south of Rio Grande do Sul State in Brazil, aimed to analyze the routine of practices of the healthcare professionals, with the integrality of the organization of practices as its reference. Data was collected via interviews with 21 members of the team and the categories Interaction and construction spaces: integrality in daily practices and Difficulties met in daily work were identified through thematic analysis. It was verified that the logic of the work is constituted in a user-centred manner; that interaction and construction spaces provide opportunities for integration between team and service users, potentializing the integrality. Despite some difficulties, such as the existence of the mental health clinic in the service, it is concluded that the practices promote integrality, as they suggest new alternatives of care, valuing the human being in suffering.
\end{abstract}

KEYWORDS: Integral assistance to health; Mental health; Health services.

\section{LA INTEGRALIDAD EN EL COTIDIANO DE LAS PRÁCTICAS EN UN CENTRO DE ATENCIÓN PSICOSOCIAL}

RESUMEN: Estudio de caso descriptivo, con abordaje cualitativo, realizado en un Centro de Atención Psicosocial I, al sur del Estado de Rio Grande do Sul, que tuvo por objetivo analizar el cotidiano de las prácticas de los profesionales y como referencial la integralidad en la organización de las prácticas. Los datos fueron recogidos por medio de entrevista a 21 integrantes del equipo y por medio del análisis temático surgieron las categorías Espacios de interacción y construcción: la integralidad en las prácticas cotidianas y Dificultades afrontadas en el cotidiano del servicio. Se verificó que la lógica del servicio se constituye de modo usuario-centrado; que espacios de interacción y construcción propician la integración entre equipo y usuarios, potencializando la integralidad. A pesar de algunas dificultades, como la existencia del ambulatorio de salud mental dentro del servicio, se concluyó que las prácticas están de acuerdo a la integralidad, ya que se proponen a la construcción de nuevas alternativas de cuidado, valorando el ser hunano en sufrimiento.

PALABRAS-CLAVE: Asistencia integral a la salud; Salud mental; Servicios de salud.

*Recorte da pesquisa "Avaliação dos CAPS da Região Sul do Brasil", financiada pelo CNPq e apoiada pelo Ministério da Saúde.

${ }^{1}$ Enfermeira. Mestre em Enfermagem. Enfermeira da Estratégia Saúde da Família da Secretaria Municipal de Saúde de Pelotas-RS.

${ }^{2}$ Enfermeira. Doutora em Enfermagem Psiquiátrica. Professora da Faculdade e do Programa de Pós-Graduação em Enfermagem da Universidade Federal de Pelotas - UFPel.

${ }^{3}$ Enfermeira. Doutora em Enfermagem. Professora da Faculdade e do Programa de Pós-Graduação em Enfermagem da UFPel.

${ }^{4}$ Enfermeira. Doutora em Enfermagem em Saúde Pública. Professora da Faculdade e do Programa de Pós-Graduação em Enfermagem da UFPel.

${ }^{5}$ Acadêmica de Enfermagem da UFPel. 


\section{INTRODUÇÃO}

Para o Ministério da Saúde a integralidade é um dos princípios mais importantes do Sistema Único de Saúde (SUS), pois a atenção à saúde deve considerar as necessidades particulares de pessoas ou de grupos específicos, mesmo que minoritários em relação à totalidade da população ${ }^{(1)}$. Na IV Conferência Nacional de Saúde Mental Intersetorial, realizada em 2010, reafirmou-se a proposta de garantir o acesso universal aos cuidados em saúde mental, assegurando-se que essas políticas atendam aos princípios do SUS, dando ênfase à integralidade e promovendo a inserção e a participação dos usuários na rede de saúde mental ${ }^{(2)}$.

Como dispositivo estratégico dessa rede tem-se os Centros de Atenção Psicossocial (CAPS), que articulados a outros setores e redes sociais buscam responder à complexidade das demandas de sujeitos portadores de transtornos mentais. Esses novos dispositivos, substitutivos ao manicômio, são serviços de atenção diária, abertos, com livre acesso aos usuários, familiares e sociedade, estando pautados no atendimento territorializado e integral. Nesses serviços, o foco do cuidado é o sujeito em sofrimento, e não unicamente a doença ${ }^{(3)}$.

Nesta perspectiva, entende-se que as práticas desenvolvidas nos CAPS devem ser guiadas pela integralidade, já que têm a característica do cuidado em liberdade, promovem a inclusão social e buscam estratégias conjuntas para superar as adversidades.

$\mathrm{O}$ agir dos profissionais a partir do eixo norteador da integralidade remete à lógica de que o serviço deve estar organizado de maneira usuário-centrada, pois ao se eleger a implementação de práticas sob o princípio da integralidade, espera-se maior capacidade na compreensão das necessidades dos sujeitos, em busca de respostas adequadas às suas demandas ${ }^{(4)}$.

Desenvolver uma prática usuário-centrada pressupõe priorizar a dimensão cuidadora do trabalho, não se centrando apenas em procedimentos, mas sim investindo em tecnologias mais relacionais ao assistir o usuário. Nesta prática, o olhar da equipe está voltado às relações acolhedoras, à retomada do diálogo, à escuta e à criação de vínculos ${ }^{(5)}$.

Pode-se refletir acerca da integralidade abordando três conjuntos de sentidos distintos a ela atribuídos. No primeiro conjunto, a integralidade, como um traço da boa medicina, implica em não restringir a compreensão do paciente ao sistema biológico que gerou o sofrimento, mas sim direcionar um olhar abrangente às necessidades dos usuários que buscam um serviço de saúde. No segundo conjunto, encontrase a integralidade como modo de arranjar as práticas, destacando-se a importância da organização contínua do processo de trabalho, quando se procura ampliar as possibilidades de compreender as necessidades de saúde da população. O terceiro conjunto de sentidos para a integralidade contempla as políticas especiais, projetadas para responder a determinado problema de saúde ou problemas que atingem certos grupos populacionais específicos. As políticas mais próximas do princípio da integralidade, neste sentido, seriam aquelas que compreendem o aspecto preventivo e assistencial simultaneamente ${ }^{(4)}$.

Dentre os três conjuntos de sentidos da integralidade elegeu-se, para o presente estudo, o segundo conjunto, a integralidade como um modo de organizar as práticas com a fundamentação teórica, pois a organização dos serviços e das práticas de saúde precisa ser pensada de forma a contemplar as reais necessidades dos usuários, estabelecendo um processo de trabalho capaz de apreender o que as pessoas querem e precisam, numa perspectiva de contratualização entre equipe e usuários ${ }^{(4)}$. $\mathrm{O}$ CAPS foi escolhido como cenário por representar um espaço no território onde práticas cotidianas podem propiciar o encontro de saberes e vivências de profissionais e usuários, em um processo de reconstrução em busca da cidadania. O objetivo da pesquisa foi analisar a integralidade no cotidiano das práticas dos profissionais no CAPS.

\section{METODOLOGIA}

Esta pesquisa se constituiu em um estudo de caso descritivo, com abordagem qualitativa dos dados. É um recorte da pesquisa de Avaliação dos CAPS da Região Sul do Brasil (CAPSUL), desenvolvida em 2006, financiada pelo Ministério da Ciência e Tecnologia através do Conselho Nacional de Desenvolvimento Científico e Tecnológico (CNPq), contemplada no Edital 07/2005 e com o apoio do Ministério da Saúde. O CAPSUL foi coordenado pela Faculdade de Enfermagem e Obstetrícia da Universidade Federal de Pelotas e desenvolvido em parceria com a Escola de Enfermagem da Universidade Federal do Rio Grande do Sul e com o Curso de Enfermagem da Universidade Estadual do Oeste do Paraná.

Foi realizada uma avaliação de serviços que se des- 
dobrou em um estudo qualitativo e outro quantitativo, nos Estados do Rio Grande do Sul, Santa Catarina e Paraná. O estudo de avaliação qualitativa utilizou a Avaliação de Quarta Geração para nortear o processo teórico-metodológico. Foram realizados cinco estudos de caso, por meio de entrevistas e observações com equipe, familiares e usuários. Já na avaliação quantitativa, foi utilizada a abordagem epidemiológica, avaliando-se estrutura, processo e resultado da atenção em saúde mental desenvolvida nos CAPS.

O presente estudo foi realizado em um CAPS I, localizado ao sul do Estado do Rio Grande do Sul. A seleção do município foi intencional, por se tratar de um CAPS que é referência para os demais da Região Sul do estado e por sua adequação às normas definidas pela Portaria n.336/GM de 2002. Também foram observados o tempo de funcionamento, a experiência do serviço e a disponibilidade dos profissionais em aderir à proposta.

Utilizou-se o banco de dados qualitativo, compreendendo as entrevistas semiestruturadas aos 21 profissionais do serviço. Compunham a equipe, no momento da pesquisa, dois funcionários dos serviços gerais, uma educadora física, três técnicos em enfermagem, três psicólogas, dois psiquiatras, duas secretárias, um motorista, quatro acompanhantes terapêuticos, uma cozinheira, uma assistente social e uma economista. A coleta de dados ocorreu no mês de outubro de 2006.

O projeto de pesquisa foi apreciado e aprovado pelo Comitê de Ética e Pesquisa da Faculdade de Medicina da UFPel (n.074/05) e todos os entrevistados assinaram o termo de consentimento livre e esclarecido. Para a garantia do anonimato, os sujeitos foram identificados pela letra " $E$ " seguida de algarismos arábicos em ordem crescente (E1, E2, etc.).

As entrevistas foram gravadas e posteriormente transcritas de forma literal, garantindo maior fidedignidade às informações. Quanto aos dados, elegeu-se a análise temática, por melhor atender à investigação qualitativa do material relacionado à saúde ${ }^{(6)}$.

Da análise dos dados deste estudo foram desveladas duas categorias: Espaços de interação e construção: a integralidade nas práticas cotidianas e Dificuldades enfrentadas no cotidiano do serviço. Dentro da primeira categoria, foi abordada a construção coletiva do projeto terapêutico do serviço e a importância do espaço encontrado na reunião de equipe. Na segunda, discorreu-se sobre o ambulatório de saúde mental e o serviço de atenção diária.

\section{RESULTADOS}

\section{Espaços de interação e construção: a integralidade nas práticas cotidianas}

As falas a seguir apontam para os espaços encontrados no CAPS que promovem a interação entre a equipe e os usuários do serviço, destacando-se a construção coletiva do projeto terapêutico e a reunião de equipe. Pelos depoimentos, observa-se que a elaboração do projeto terapêutico de forma coletiva pode potencializar a integralidade:

[...] quem faz o projeto terapêutico é uma equipe multidisciplinar [...] a gente foi construindo aos pouquinhos conforme a necessidade e a realidade [...] o projeto terapêutico tem que ser uma coisa flexivel onde possa ser construído, mas ao mesmo tempo modificado de acordo com a realidade que a gente tem no momento. (E1)

[...] hoje nós estamos tentando trabalhar numa cogestão [...] as mudanças do projeto terapêutico vêm até pela maturidade da equipe [...]. Há muita flexibilidade, não existe alguém que dita a regra e todos seguem [...] existe sim um grupo de pessoas que onde um tem uma ideia diferente da outra é colocado em reunião e avaliado [...] isso pode vir da faxineira até da coordenação [...]. (E3)

A participação dos usuários na construção do projeto terapêutico e a preocupação em inserí-los em atividades que realmente tragam benefícios são fatos apontados nos seguintes discursos:

[...] o usuário que procura o CAPS está tendo a oportunidade de construir junto com a gente aquilo que ele mais gosta. De que adianta ele entrar no CAPS e ter que fazer a atividade como uma obrigação? [...] essa atividade tem que ser prazerosa, tem que contribuir para a melhoria dele. [...] a construção do plano, juntamente com o usuário, é uma das coisas mais importantes. (E8)

[...] a coisa mais importante no projeto terapêutico é conseguir cuidar no território, sem contar com o hospital psiquiátrico [...] a integralidade da ação que trabalha promoção, prevenção, tratamento e reabilitação. O projeto terapêutico tem que estar envolvido na integralidade. (E12) 
A reunião de equipe, espaço de planejamento e organização do plano de trabalho, aparece como um momento de interação e construção para os diversos profissionais do serviço:

A reunião de equipe é uma coisa que fortalece a gente, ajuda uns a segurar a barra dos outros [...] as pessoas podem se despir de formalidades técnicas para ver as coisas de forma mais abrangente e não reducionista, utilizar mais tecnologias leves que dependem do relacionamento humano. [...] fala-se um discurso de integralidade, isso tem que ser sentido, tem que ser vivenciado [...]. (E2)

Antes eu não participava das reuniões, dai me chamaram para eu começar a participar. Eu comecei a participar, até para conhecer mais os casos de cada um, para quando vierem conversar comigo eu saber o que responder, estar inteirada [...]. (E11)

\section{Dificuldades enfrentadas no cotidiano do serviço}

Alguns profissionais destacaram dificuldades relacionadas à localização do ambulatório de saúde mental, que se encontra inserido no CAPS. A seguir, alguns depoimentos:

[...] uma coisa que me incomoda muito dentro do serviço é a questão ambulatorial, por que ela se confunde muito, por que para ter um ambulatório aqui dentro que é de demanda por receita, deveria estar separado de um serviço como o nosso, de atenção diária, ter uma equipe ambulatorial para fazer isso num outro local [...]. E a gente perde um tempo precioso em atender os nossos usuários, presta um outro cuidado. (E2)

[...] minha crítica sempre foi no sentido de nós ficarmos envolvidos muito tempo com a parte do ambulatório e não conseguirmos dar mais atenção aos usuários. $O$ atendimento ambulatorial também é importante, mas eu acho que prejudica, tem uma demanda muito grande de acolhimento. Eu acho que o bom atendimento do CAPS é aquele que tu sentas, tomas café junto, estás na oficina junto, vais na horta [...]. (E10)

Vale ressaltar algumas críticas dos profissionais no âmbito da organização do serviço, principalmente aquelas relacionadas aos poucos recursos humanos disponíveis e seus horários, o que dificulta o contato com os usuários:
O ambulatório de psicologia até hoje está sendo muito complicado. Tem pessoas que não são do nosso sistema, mas vêm aqui fazer psicoterapia e aí fica pouco pessoal da psicologia para fazer atendimentos aos nossos usuários para as demandas mais pontuais. (E2) Eu acho que tem muita demanda, deixam de atender o nosso paciente lá em baixo. [...] às vezes tem uns da equipe que dizem: "ai, eu nem tive tempo hoje de descer para falar com o fulano". (E16)

Embora a inserção do ambulatório no espaço do CAPS seja considerada problemática pela maior parte dos entrevistados, registra-se uma fala que assinala o valor desse serviço na rede de saúde do município:

O ambulatório para nós é muito importante [...] ele é muito grande. Hoje em dia acaba nos tirando muito da possibilidade de dar conta de todas as demandas que necessitariam de cuidado. Só que o ambulatório vai ter um papel muito importante quando mudar a gestão de saúde do território, no momento que o município passar a adotar o Programa de Saúde da Família, aí desafoga. Nós vamos achar, a partir daí, uma abertura para a entrada da saúde mental na rede básica. (E12)

Observa-se que a estrutura física do prédio também foi apontada como fator capaz de dificultar a comunicação dos profissionais entre si e com os usuários, já que o prédio tem dois andares:

O funcionamento da casa poderia ser bem melhor se a gente não estivesse aqui, estivesse numa casa mais espaçosa [...] ficam uns em cima e outros embaixo. Acho que a gente está muito encerrada. O pessoal também se divide muito, se fosse uma casa mais parelha todo mundo ficava mais junto. (E4)

\section{DISCUSSÃO}

\section{Espaços de interação e construção: a integralidade nas práticas cotidianas}

Esta categoria discute a percepção dos profissionais em relação aos espaços existentes no CAPS que podem propiciar a interação entre equipe e usuários. A organização das práticas de saúde precisa ser pensada de forma a contemplar as necessidades dos usuários, estabelecendo um processo de trabalho capaz de apreender o que as pessoas querem e precisam, numa perspectiva de contratualização entre equipe e usuários. 
Os discursos demonstraram que a elaboração do projeto terapêutico do serviço pela equipe interdisciplinar, de forma flexível, coletiva e compartilhada, com a participação dos usuários na construção das atividades propostas, pode ser considerada um fator potencializador da integralidade.

Os projetos terapêuticos que surgem na perspectiva da integralidade resultam do diálogo e da negociação contínua entre usuários e profissionais. A principal característica a ensejar esse diálogo é a habilidade para compreender o contexto específico dos distintos encontros, considerando os conhecimentos técnicos, bem como os trazidos pelo usuário, a partir de suas expectativas, seus receios, suas dores e seus desejos ${ }^{(7)}$. Nesses projetos tem-se uma importante ferramenta para o desenvolvimento do cuidado ao usuário com transtorno mental, já que serve de eixo de referência para orientar as ações dos profissionais do serviço na busca da reabilitação psicossocial ${ }^{(8)}$.

Oferecer ao usuário a atenção psicossocial na perspectiva da integralidade implica não enxergar somente o transtorno mental, mas também concebê-lo como sujeito de sua própria história, o qual possui sonhos, medos, expectativas e necessidades que não apenas as de saúde, para que possa novamente conduzir sua vida.

Este modelo de atenção psicossocial, que adota o território como espaço de ação, tem se configurado como ambiente substitutivo à internação de longa permanência, já que prioriza a participação do usuário e familiares no tratamento, facilitando a reinserção social por meio de projetos terapêuticos que contemplam atividades variadas, promovendo o exercício da cidadania, lazer e inclusão. Para tanto, espera-se que os CAPS façam a crítica ao universo manicomial e sejam lugar de constituição de práticas alternativas e substitutivas ${ }^{(9)}$. OCAPS é o centro de uma nova clínica, que gera autonomia e estimula o usuário a responsabilizar-se por sua vida e a opinar no curso do seu tratamento ${ }^{(10)}$.

Ainda nesta categoria, observou-se que a existência de um espaço para o planejamento e organização do processo de trabalho da equipe, encontrado na reunião dos profissionais, permite a oferta de um cuidado mais integral e resolutivo, se configurando num espaço de interação e construção. A articulação entre os saberes dos vários profissionais possibilita a construção de um processo de trabalho coletivo, considerando a complexidade dos indivíduos.

O trabalho em equipe ocorre por meio da interação entre os diversos atores, trabalhadores com diferentes formações; a maior potencialidade está justamente no desafio de se articularem, apesar dos diferentes campos de saber, para produzir ações saúde. Orientar as práticas pela integralidade significa romper com as hierarquias profissionais e a compartimentalização do cuidado, pressupondo um saber-fazer comum ${ }^{(11)}$.

A reunião de equipe é aqui compreendida como um ponto importante que pode intensificar a integralidade no cotidiano do CAPS. Perceberam-se relações horizontais entre os membros da equipe, eliminando-se a hierarquização das relações, havendo reconhecimento e valorização de todos os campos de saberes, e contribuindo para um processo de trabalho direcionado às necessidades do usuário.

\section{Dificuldades enfrentadas no cotidiano do serviço}

Entre as dificuldades encontradas no cotidiano do serviço destacou-se a questão do ambulatório de saúde mental, por este situar-se nas dependências do CAPS. Para a maioria dos profissionais o ambulatório de psicologia e psiquiatria deveria estar locado em espaço independente, já que sua finalidade é responder a demandas diferentes do serviço de atenção diária. É compreensível a crítica de alguns profissionais em relação ao funcionamento de ambos os serviços dentro do mesmo espaço físico, bem como a utilização dos mesmos recursos humanos. Cabe salientar que esses dois serviços de saúde mental possuem lógicas e processos de trabalho distintos.

$\mathrm{O}$ atendimento em saúde mental prestado em ambulatório especializado compreende um conjunto diversificado de atividades, sendo que os critérios de hierarquização e regionalização da rede, bem como a população de referência, são estabelecidos pelo gestor local ${ }^{(12)}$. Já os CAPS são estruturas que viabilizam o cuidado com base na atenção psicossocial, entendida como um conjunto de práticas com características próprias, especialmente quanto à constituição das equipes, à maneira como avaliam seu objeto de intervenção, às formas de organização institucional e ao modo de se relacionar com os usuários ${ }^{(13)}$.

Os CAPS são estabelecimentos designados para acolher os usuários com transtornos mentais, encorajar sua integração social e familiar e ampará-los em suas ações em direção à autonomia. Seu atributo essencial é a busca da integração em um ambiente social e cultural real, reconhecido como seu território, um espaço da cidade onde se movimenta a vida cotidiana de usuários e seus familiares ${ }^{(14)}$. 
A existência do ambulatório dentro do CAPS muitas vezes compromete o horário de alguns profissionais, diminuindo o tempo dedicado aos usuários, ficando menos espaços para o contato diário, essencial na socialização, ainda mais se tratando de um serviço que preza o cuidado e o contato como pontos importantes do tratamento. Contudo, houve quem evidenciasse a importância desse serviço, no sentido de estar futuramente articulado à rede básica de saúde do município, estabelecendo parcerias.

A compreensão de rede e território é essencial para o entendimento do papel estratégico dos serviços de saúde mental, sendo que isto se ajusta também à sua relação com a rede básica de saúde. A Reforma Psiquiátrica caminha no sentido de conduzir o centro do cuidado para fora do hospital, em direção à comunidade, e estes serviços são dispositivos estratégicos desse movimento. A rede básica de saúde é o local privilegiado para a constituição de uma nova lógica de atendimento em saúde mental ${ }^{(14)}$.

Neste sentido, pode-se afirmar que na atenção básica há condições para desenvolver-se duas importantes ações em saúde mental. A primeira está em detectar as queixas referentes ao sofrimento psíquico e promover uma escuta qualificada para essa problemática. A segunda implica nas formas de abordar os agravos encontrados, proporcionando cuidados na própria atenção básica, articulando-se com o serviço especializado ${ }^{(15)}$.

Outra dificuldade que apareceu atrelada à presença do ambulatório no serviço foi a estrutura física do prédio, que possui dois andares. Segundo foi manifestado, este fato dificulta o encontro e o convívio dos profissionais entre eles e com os usuários, já que as atividades acontecem em ambientes distintos, ficando uns e outros privados daquele contato essencial na socialização dos usuários.

Sem desconsiderar o discurso dos profissionais, importa sublinhar que a conformação física do prédio não é o único fator responsável pelo distanciamento entre profissionais e usuários. Certamente a cultura dos atendimentos centrados na consulta individual e o apreço pelas salas fechadas, herança do modelo biomédico, podem contribuir para a diminuição do contato entre os profissionais e os usuários.

\section{CONSIDERAÇÕES FINAIS}

Os depoimentos recolhidos revelam um processo de trabalho arquitetado através da agregação dos diversos campos de saberes e compartilhado com os usuá- rios, estando a relação da equipe baseada no respeito ao outro, considerando sua participação na produção do cuidado. Identificou-se que os profissionais valorizam a diversidade dos olhares, cientes da exigência de ampliar as percepções em relação aos usuários, mesmo porque o que conduz à integralidade no cotidiano das práticas é a pluralidade dos modos de cuidar ao invés do simples tratar, que pode levar ao reducionismo.

O estudo também demonstrou que existe o entendimento, por parte dos profissionais do serviço, da necessidade de um outro modelo de atenção, baseado no cuidar e não no tratar, cuidado esse mais humano e emancipatório.

Apesar das dificuldades enfrentadas no cotidiano de trabalho, os atores sociais envolvidos com a história desse CAPS buscaram alternativas para que o leque de possibilidades oferecidas aos usuários pudesse ser ampliado, com criatividade e trabalho em equipe. Os achados deste estudo refletem comprometimento, pois os profissionais oferecem um cuidado integral e humanizado ao portador de transtorno mental, visando à conquista de sua liberdade e autonomia.

Em relação às limitações desta pesquisa, de abordagem qualitativa, admite-se que não é possível generalizar os resultados encontrados no CAPS estudado para outros serviços, já que este foi escolhido intencionalmente por se ajustar a algumas características, como adequação à Portaria n. 336/02, disponibilidade dos profissionais em participar da pesquisa, experiência e tempo de funcionamento. Práticas pautadas na integralidade, evidenciadas na pesquisa, correspondem a uma realidade bastante específica, mas também desejável aos demais serviços de saúde.

A discussão sobre a integralidade no universo do CAPS certamente não se esgota neste estudo, mesmo porque práticas cotidianas dentro desse serviço estão em constante transformação e renovação, longe de serem estáticas. Viabilizar a integralidade no cotidiano das práticas requer a busca da adequação das relações entre trabalhadores e usuários, diversificando-se as formas de cuidar e dispondo-se a uma nova proposta de trabalho.

\section{REFERÊNCIAS}

1. Ministério da Saúde (BR). Secretaria Executiva. Sistema Único de Saúde (SUS): princípios e conquistas. Brasília: Ministério da Saúde; 2000.

2. Ministério da Saúde (BR). Sistema Único de Saúde. Conselho Nacional de Saúde. Comissão Organizadora da IV CNSMI. Relatório Final da IV Conferência 
Nacional de Saúde Mental - Intersetorial. Brasília: Conselho Nacional de Saúde/Ministério da Saúde; 2010.

3. Mielke FB, Kantorski LP, Jardim VMR, Olschowski A. Avaliação de um serviço substitutivo em saúde mental. Cogitare Enferm. 2009;14(1):52-8.

4. Mattos RA. Os sentidos da integralidade: algumas reflexões acerca de valores que merecem ser defendidos. In: Pinheiro RP, Mattos RA, organizadores. Os sentidos da integralidade na atenção e no cuidado à saúde. Rio de Janeiro: UERJ, IMS, ABRASCO; 2001. p.39-64.

5. Franco TB, Merhy EE. A produção imaginária da demanda e o processo de trabalho em saúde. In: Pinheiro RP, Mattos RA, organizadores. Construção social da demanda: direito à saúde, trabalho em equipe, participação e espaços públicos. Rio de Janeiro: UERJ, IMS, CEPESC, ABRASCO; 2005. p.181-94.

6. Minayo MCS. Pesquisa social: teoria, método e criatividade. Petrópolis: Vozes; 1998.

7. Amarante PDC. Saúde mental e atenção psicossocial. Rio de Janeiro: Fiocruz; 2007.

8. Kantorski LP, Bielemann VLM, Clasen BL, Padilha MAS, Bueno MEN, Heck RM. A concepção dos profissionais acerca do projeto terapêutico de Centros de Atenção Psicossocial-CAPS. Cogitare Enferm. 2010;15(4):659-66.

9. Merhy EE. O CAPS e seus trabalhadores: no olho do furacão antimanicomial. Alegria e alívio como dispositivos analisadores. In: Merhy EE, Amaral H, organizadores. A reforma psiquiátrica no cotidiano II. São Paulo: Hucitec; 2007. p.55-66.

10. Ministério da Saúde (BR). Secretaria de Atenção à Saúde. DAPE. Coordenação Geral de Saúde Mental. Reforma psiquiátrica e política de saúde mental no Brasil. Documento apresentado à Conferência Regional de Reforma dos Serviços de Saúde Mental: 15 anos depois de Caracas. OPAS. Brasília: Ministério da Saúde; 2005.

11. Bonaldi G, Gomes RS, Louzada APF, Pinheiro R. O trabalho em equipe como dispositivo de integralidade: experiências cotidianas em quatro localidades brasileiras. In: Pinheiro R, Mattos RA; Barros MEB, organizadores. Trabalho em equipe sob o eixo da integralidade: valores, saberes e práticas. Rio de Janeiro: IMS, CEPESC, ABRASCO; 2007. p.53-72.

12. Ministério da Saúde (BR). Portaria n. 224, de 29 de janeiro de 1992. Dispõe sobre diretrizes e normas para o atendimento ambulatorial/hospitalar em saúde mental.
Diário Oficial da República Federativa do Brasil, Brasília, 30 jan. 1992. Seção 1:1160.

13. Costa-Rosa A. O modo psicossocial: um paradigma das práticas substitutivas ao modo asilar. In: Amarante PDC, organizador. Ensaios: subjetividade, saúde mental, sociedade. Rio de Janeiro: FIOCRUZ; 2000. p.141-68.

14. Ministério da Saúde (BR). Secretaria de Atenção à Saúde. Departamento de Ações Programáticas Estratégicas. Saúde mental no SUS: os centros de atenção psicossocial. Brasília: Ministério da Saúde; 2004.

15. Tanaka OY, Ribeiro EL. Ações de saúde mental na atenção básica: caminho para ampliação da integralidade da atenção. Cienc Saude Colet. 2009;14(2):477-86. 